\title{
ON POLE-PLACEMENT CONTROLLERS FOR LINEAR TIME-DELAY SYSTEMS WITH COMMENSURATE POINT DELAYS
}

\author{
M. DE LA SEN
}

Received 12 December 2003 and in revised form 18 May 2004

We investigate the exact and approximate spectrum assignment properties associated with realizable output-feedback pole-placement-type controllers for single-input singleoutput linear time-invariant time-delay systems with commensurate point delays. The controller synthesis problem is discussed through the solvability of a set of coupled Diophantine equations of polynomials. An extra complexity is incorporated in the above design to cancel extra unsuitable dynamics being generated when solving the above Diophantine equations. Thus, the complete controller tracks any arbitrary prefixed (either finite or delay-dependent) closed-loop spectrum. However, if the controller is simplified by deleting the above-mentioned extra complexity, then robust stability and approximated spectrum assignment are still achievable for a certain sufficiently small amount of delayed dynamics. Finally, the approximate spectrum assignment and robust stability problems are revisited under plant disturbances if the nominal controller is maintained. In the current approach, the finite spectrum assignment is only considered as a particular case of the designer's choice of a (delay-dependent) arbitrary spectrum assignment objective.

\section{Introduction}

Time-delay systems have received an increasing interest in the last years (see, for instance, $[2,3,4,5,6,7,12,13,15,17,18,20,21]$ since, apart from their inherent theoretical interest, they are also of interest in practical applications like, for instance, transmission lines, dynamics of fluids, or population growth rules $[3,4,18]$. One of the main characteristics of such systems is that they are infinite dimensional $[4,6,7,12,13,17]$, making the controller design more complex than for the delay-free case $[2,6,12,13,15,17,20,21]$. Closed-loop stabilization may be achieved through memoryless controllers (see, for instance, $[13,20])$ when the plant delayed dynamics is sufficiently small. However, the stabilization and spectrum assignment cannot be achieved, in general, for any amount of delayed dynamics by delay-free controllers (see $[6,17]$ and the references therein). Generally speaking, there are two main groups of techniques used for the controller synthesis. 
One of them consists of designing a stabilizing controller for the delay-free plant dynamics while considering the unsuitable combined effects caused by the interaction of the controller and the delayed dynamics as a robustness problem $[2,10,17,20,23]$. Another group of design techniques, including those of pole-placement type, consists of synthesizing controllers for the whole plant, taking into account its delayed dynamics in the design itself $[6,15,17]$. In this paper, the synthesis of an output-feedback controller with a particular structure which is based on pole placement is focused on. The obtained controller has a transfer function structure similar to that of the plant, that is, a quotient of quasipolynomials. It is proved that the controller synthesis problem is solvable, in general, with a realizable delay-dependent controller for any prefixed (either finite or delay-dependent) spectrum if the (delay-dependent) plant transfer function $P(s)$ and that obtained as a particular case when neglecting all the delayed dynamics, namely $P_{0}(s)$, are both cancellation free. The controller synthesis consists of two parts. The first one consists of the solution of a finite set of nested Diophantine equations of polynomials, all of which being sequentially solvable if and only if $P_{0}(s)$ has no zero-pole cancellation. This part of the design sets a part of the controller numerator and denominator quasipolynomials while generating an extra unsuitable dynamics in the closed-loop spectrum that is inherent in the proposed synthesis method. The second part consists of incorporating into the design an extra controller complexity to cancel the above-mentioned unsuitable dynamics so that the complete controller sets any arbitrary prefixed (either finite or delay-dependent) closed-loop spectrum. It is proved that a prefixed approximate closedloop spectrum is obtained even if the plant possesses parametrical and/or unmodeled dynamics disturbances to some extent of tolerance while the nominal controller is kept in operation. However, if the controller is simplified by deleting the second phase of the design, then robust stability and approximated spectrum assignment are still achievable for a sufficiently small amount of plant delayed dynamics. The paper is organized as follows. Section 2 deals with the controller synthesis problem for exact spectrum assignment. The approximate spectrum assignment as well as the robust internal stability are investigated in Section 3 under low-complexity controllers for sufficiently small amounts of delayed dynamics. Some design examples are also included related to the achievement of exact and approximate spectrum assignments with the proposed methods. Finally, conclusions end the paper.

\section{Controller synthesis problem}

2.1. Plant. Consider the linear and time-invariant single-input single-output plant with commensurate point delays:

$$
\begin{gathered}
\dot{x}(t)=\overline{\mathbf{A}}_{0} x(t)+\sum_{i=1}^{q_{0}} \overline{\mathbf{A}}_{i} x(t-i h)+b u(t), \\
y(t)=c^{T} x(t)+d u(t),
\end{gathered}
$$

where $h \geq 0$ is the basic delay and $h_{i}=i h\left(i=\overline{1, q_{0}}\right)$ are the commensurate point delays, $\overline{\mathbf{A}}_{i} \in \mathbb{R}^{n \times n}\left(i=\overline{0, q_{0}}\right)$, and $b, c \in \mathbb{R}^{n}, d \in \mathbb{R}$ parametrize the system. The plant is 
strictly proper (biproper) if and only if $d=0(d \neq 0)$. The signals $u(t) \in \mathbb{R}, y(t) \in \mathbb{R}$, and $x(t) \in \mathbb{R}^{n}$ are the scalar input and output and the state vector, respectively. The initial condition of (2.1a) is as follows: $\varphi:\left[-q_{0} h, 0\right] \cap \mathbb{R} \rightarrow \mathbb{R}^{n}$ is a continuous function with (possibly) isolated bounded discontinuities on a subset of $\left[-q_{0} h, 0\right] \cap \mathbb{R}$ of zero measure. The transfer function of (2.1) is defined in a standard way by using Laplace transforms of the output and input as $P(s)=[Y(s) / U(s)]_{\varphi \equiv 0}$, thus leading to

$$
P(s)=\frac{B(s)}{A(s)}=c^{T}\left(s I-\sum_{i=0}^{q_{0}} \overline{\mathbf{A}}_{i} e^{-i h s}\right)^{-1} b+d,
$$

where $A(s)$ and $B(s)$ are quasipolynomials in the indeterminate $s$ defined by

$$
\begin{aligned}
A(s) & =\operatorname{det}\left(s I-\sum_{i=0}^{q_{0}} \overline{\mathbf{A}}_{i} e^{-i h s}\right)=\sum_{i=0}^{q} A_{i}(s) e^{-i h s} \\
& =\sum_{i=0}^{n} A_{i}^{*}\left(e^{-h s}\right) s^{i}=\sum_{i=0}^{q} \sum_{k=0}^{n} a_{i k} s^{k} e^{-i h s}, \\
B(s) & =c^{T} \operatorname{Adj}\left(s I-\sum_{i=0}^{q_{0}} \overline{\mathbf{A}}_{i} e^{-i h s}\right) b+d A(s) \\
& =\sum_{i=0}^{q^{\prime}} B_{i}(s) e^{-i h s}=\sum_{i=0}^{m} B_{i}^{*}\left(e^{-h s}\right) s^{i}=\sum_{i=0}^{q^{\prime}} \sum_{k=0}^{m} b_{i k} s^{k} e^{-i h s}
\end{aligned}
$$

with $q$ and $q^{\prime}$ being integers satisfying $q^{\prime} \leq q \leq q_{0} n$. For exposition simplicity, it is assumed with no loss in generality that $q^{\prime}=q$. Otherwise, $(2.3 \mathrm{~b})$ still applies by zeroing the necessary polynomials $B_{(\cdot)}$.

$B_{i}(s)=\sum_{k=0}^{m_{i}} b_{i k} s^{k}$ and $A_{i}(s)=\sum_{k=0}^{n_{i}} a_{i k} s^{k}$ are polynomials of respective degrees $m_{i}$ and $n_{i}(i=\overline{0, q})$ with $m_{i} \leq m_{0}=m \leq n$ and $n_{i} \leq n_{0}=n$ for $i=\overline{0, q}$ with $m=n$ if and only if $d \neq 0$ in (2.1b), that is, the plant is not a strictly proper plant, and $m \leq n-1$ otherwise. Note that $n=n_{0} \geq \operatorname{Max}\left(m, \operatorname{Max}_{1 \leq i \leq q}\left(n_{i}, m_{i}\right)\right)$ since the transfer function (2.2)-(2.3) obtained from (2.1) is realizable. After normalization of the coefficients of the transfer function if necessary, $A_{0}(s)$ may be chosen as monic; that is, $a_{0 n}=1$. Alternative polynomials $B_{i}^{*}\left(e^{-h s}\right)$ and $A_{i}^{*}\left(e^{-h s}\right)$ are defined in the same way leading to an equivalent description of (2.1)-(2.2).

2.2. Controller parametrization and control law. The members of the class of outputfeedback single-input single-output controllers to be synthesized have the general transfer function structure

$$
\begin{aligned}
K_{v}(s) & =\frac{S(s)}{R(s)}=\frac{\sum_{i=0}^{v} S_{i}(s) e^{-i h s}}{\sum_{i=0}^{v-1} R_{i}(s) e^{-i h s}+R_{v}(s)} \\
& =\frac{D_{v}(s)\left(\sum_{i=0}^{v} \sum_{l=0}^{m_{i}^{\prime}} s_{i \ell} s^{l} e^{-i h s}\right)}{D_{v}(s)\left(\sum_{i=0}^{v-1} \sum_{l=0}^{r_{i}^{\prime}} r_{i \ell} s^{l} e^{-i h s}\right)+N_{\nu}(s) e^{-v h s}}
\end{aligned}
$$


for some integer $v$ with $q \geq v \geq 1$, where

$$
S_{i}(s)=\sum_{l=0}^{m_{i}^{\prime}} s_{i l} s^{l}, \quad R_{i}(s)=\sum_{l=0}^{n_{i}^{\prime}} r_{i l} s^{l}
$$

are polynomials of respective degrees:

(i) $\partial S_{i}=m_{i}^{\prime} ; \partial R_{i}=n_{i}^{\prime}(i=\overline{0, v-1})$;

(ii) $S_{v}(s)$ is a polynomial of degree $\partial S_{v}=m_{v}^{\prime} \leq n-1$;

(iii) $R_{v}(s)=N_{v}(s) / D_{v}(s)$ is, in general, a proper or improper rational function defined as a quotient of quasipolynomials to be specified later.

It is apparent that the controllers of transfer function (2.4) are proper if $n^{\prime}=n_{0}^{\prime} \geq$ $\operatorname{Max}\left(\operatorname{Max}_{1 \leq i \leq v-1}\left(n_{i}^{\prime}\right), \operatorname{Max}_{0 \leq i \leq v-1}\left(m_{i}^{\prime}\right)\right)$ with $m^{\prime}=m_{0}^{\prime}$ for any proper or improper $R_{v}(s)$ and any parametrization. The control law obtained from the above controllers becomes

$$
u(t)=-\frac{S(D)}{R(D)}\left(y(t)-y^{*}(t)\right)
$$

with $y^{*}(t)$ being any given uniformly bounded reference signal and $D=d / d t$ is the timederivative operator (formally equivalent to the Laplace operator).

2.3. Control objective. From (2.2), and (2.4)-(2.6), the closed-loop characteristic quasipolynomial becomes

$$
A(s) R(s)+B(s) S(s)=\hat{A}_{m}(s)
$$

where $\hat{A}_{m}(s)=0$ specifies the closed-loop characteristic modes. The subsequent discussion relies on the conditions of the existence of controllers of transfer function (2.4) to accomplish the identity (2.7) for any prescribed strictly Hurwitzian quasipolynomial $\hat{A}_{m}(s)=\sum_{l=0}^{q_{m}} \hat{A}_{m l}(s) e^{-l h s}$ with $\hat{A}_{m i}(s)=\sum_{l=0}^{n_{m_{i}}} \hat{a}_{m i l}(s) s^{l}$ for any given integer $q_{m}$ satisfying $0 \leq q_{m} \leq v+q, q \geq v \geq 1$. If $\hat{A}_{m 0}(s)$ is strictly Hurwitzian and $\hat{A}_{m l}(s)=0$ for all $l=\overline{1, q+v}$, then a suitable stable closed-loop spectrum is finite and delay-independent. If $\hat{A}_{m l}(s)$ is not identically zero for all $l=\overline{1, q+v}$, then the spectrum is infinite and delay-dependent. Note that the proposed design is very flexible, contrary to previous results in the literature $[6$, $12,13,16,17]$, to achieve prefixed suitable either delay-independent or delay-dependent closed-loop spectrum. This more general problem statement may be beneficial in some applications where the suitable closed-loop spectrum is reallocated, but still delaydependent, due to the intrinsic delayed nature of the original open-loop plant $[3,4,18]$. 
The expansions of (2.7) in powers of $e^{-h s}$ yields

$$
\begin{aligned}
A(s) R(s)+B(s) S(s)= & \sum_{l=0}^{v+q} \sum_{i=\operatorname{Max}(0, l-v)}^{\operatorname{Min}(l, q)}\left[A_{i}(s) R_{l-i}(s)+B_{i}(s) S_{l-i}(s)\right] e^{-l h s} \\
= & \left\{\sum_{\ell=0}^{v-1} \sum_{i=\operatorname{Max}(0, l-v)}^{\operatorname{Min}(l, q)}\left[A_{i}(s) R_{l-i}(s)+B_{i}(s) S_{l-i}(s)\right] e^{-l h s}\right\} \\
& +\left\{\sum_{\ell=v}^{v+q} \sum_{i=\operatorname{Max}(0, l-v)}^{\operatorname{Min}(l, q)}\left[A_{i}(s) R_{l-i}(s)+B_{i}(s) S_{l-i}(s)\right] e^{-l h s}\right\} \\
= & \left\{\hat{A}_{m}^{*}(s)\right\}+\{0\},
\end{aligned}
$$

where $\hat{A}_{m}^{*}(s)=\sum_{l=0}^{v-1} A_{m l}(s) e^{-l h s}$ defines a suitable closed-loop spectrum with $\partial \hat{A}_{m i}(s)=$ $n_{m i}$. If $v=1$, then the desired spectrum is in particular delay-independent and finite. The solvability of (2.8) for any given integer $v$ satisfying $q \geq v \geq 1$ is decomposed into the combined solvability of the two corresponding identities resulting from equating the corresponding terms in key brackets at both sides of the last equality. The first identity holds if there exist pairs of polynomials $\left(R_{i}(s), S_{i}(s)\right), i=\overline{0, v-1}$, a polynomial $S_{v}(s)$, and a rational complex function $R_{v}(s)$ which satisfy

$$
\begin{gathered}
A_{0}(s) R_{i}(s)+B_{0}(s) S_{i}(s)=\hat{A}_{m i}(s)-\sum_{l=1}^{i}\left(A_{l}(s) R_{i-l}(s)+B_{l}(s) S_{i-l}(s)\right), \quad i=\overline{0, v-1}, \\
\Delta \hat{A}_{m}^{*}(s)=\sum_{\ell=v}^{v+q} \sum_{i=\operatorname{Max}(0, l-v)}^{\operatorname{Min}(l, q)}\left[A_{i}(s) R_{l-i}(s)+B_{i}(s) S_{l-i}(s)\right] e^{-l h s}=0 .
\end{gathered}
$$

The second identity is ensured after canceling the unsuitable terms contained in $\Delta \hat{A}_{m}^{*}(s)$ in the closed-loop spectrum by appropriate choice of a rational complex function $R_{\nu}(s)$ with the polynomial $S_{\nu}(s)$ being arbitrary of degree not exceeding $(n-1)$. In the case when $n=m$ (which implies $d \neq 0$ ), the inverse system of (2.1) is realizable and the controller might be alternatively synthesized by choosing $R_{v}(s)$ as a prescribed polynomial or quasipolynomial with the given degree constraints for $(2.1)$ and $S_{v}(s)$ being a rational complex function of denominator $B(s)$. The controller synthesis problem is now decomposed in the combined solution of both (2.9)-(2.10). The method consists of first solving (2.8) irrespective of (2.9) in the polynomial pairs of polynomials $\left(R_{i}(s), S_{i}(s)\right), i=\overline{0, v-1}$, which is performed sequentially from $i=0$ to $i=v-1$. Then (2.10) is solved in $\left(R_{v}(s), S_{v}(s)\right)$ after substitution of the above solutions. Usually, $R_{v}(s)$ is a rational function and $S_{v}(s)$ is a polynomial.

2.4. Controller synthesis for closed-loop spectrum assignment. The three following additional assumptions are made.

(A1) The quasipolynomials $A(s)$ and $B(s)$ have no common zeros.

(A2) The polynomials $A_{0}(s)$ and $B_{0}(s)$ have no common zeros. 
(A3) The unforced time-delay system (2.1) is asymptotically stable if $n>m$, that is, $A(s)$ has all its zeros in Res $<0$. Either system (2.1) or its inverse is asymptotically stable when $n=m$; that is, either $A(s)$ or $B(s)$ has all its zeros in Res $<0$ if $d \neq 0$ in (2.2).

(A1) means that system (2.1) is spectrally controllable and spectrally observable [8, 16]. This is an obvious requirement for the existence of a control law for prefixed spectrum assignment via output-feedback controllers. This is a more stringent condition than the spectral stabilizability required for the existence of a stabilizing control law $[1,2,9,14,15]$. Note in (2.8)-(2.9) that if (A1) fails, then the common zeros of $A(s)$ and $B(s)$ should be included as zeros of $\hat{A}_{m}(s)$ so that the closed-loop spectrum is not completely of the designer's free choice. (A2) is equivalent to the particular delay-free system obtained from (2.1) for $\bar{A}_{i}=0, i=\overline{1, q}$ being completely controllable and observable. This condition is needed as inherent in the method proposed to solve the part of the controller synthesis problem related to fix $\hat{A}_{m}^{*}(s)$. In particular, it is required to solve sequentially the Diophantine equations of polynomials in (2.9). (A3) is needed due to the fact that the controller proposed generates a closed-loop zero-pole cancellation of the plant poles (or zeros) which are removed in this way from the closed-loop spectrum. This follows from the fact that $R_{\nu}(s)$ cancels the plant poles (zeros) in order to remove the spectrum included in $\hat{A}_{m}(s)$ that is not in $\hat{A}_{m}^{*}(s)$ as will be discussed later on. If system (2.1) is not asymptotically stable but is stabilizable, it may be first stabilized via some stabilizing controller to then apply the proposed technique for spectrum assignment to the stabilized system. An important practical question is the following which is obvious by simple inspection. If (A2) holds (i.e., the system without delayed dynamics, or when the base delay tends to infinity, is controllable and observable), then (A1) holds (i.e., the overall delay system is spectrally controllable and observable) for almost all values of the basic delay $h$ for each given nonzero parametrization of the delayed dynamics. Thus, it is sufficient in practice to test that (A2) holds to guarantee that (A1) holds as well for almost all values of the base delay $h$. Note that $B_{0}(s) / A_{0}(s)$ is the transfer function of the plant as the delays tend to infinity. Thus, it follows that Assumptions (A1)-(A2) are not mutually independent from each other since if (A1) holds, then (A2) holds for all $h \geq 0$ and as $h \rightarrow \infty$. Also, if (A2) holds, then (A1) holds for almost every finite base delay $h \geq 0$ or as this one tends to infinity. Both assumptions are not exactly equivalent since (A2) is a condition which excludes a set of zero-pole cancellations from a potential finite number of them while (A2) excludes any cancellation from infinitely many possible ones. On the other hand, Assumptions (A1)-(A2) have a clear technical role in the proof of Theorem 2.1 related to the solvability of Diophantine equations of quasipolynomial and polynomials, respectively. While (A1) concerns the whole description of the plant involving point delays, (A2) (which is automatically guaranteed if (A1) holds) is concerned with the solvability of a nested set of diophnatine equations whose coefficient polynomials are $A_{0}(s)$ and $B_{0}(s)$.

\subsection{Main result of this section}

Theorem 2.1 (exact spectrum assignment and closed-loop stability). The following items hold.

(i) If (A1) holds, then there exist infinitely many polynomial pairs $\left(R_{i}(s), S_{i}(s)\right)$ which satisfy the $v$ nested Diophantine equations of polynomials independent of the base delay 
$h \geq 0$ as in (2.9). Furthermore, if $n_{m 0} \geq 2 n-1$, then there is at least a solution $\left(R_{i}(s), S_{i}(s)\right)$, $i=\overline{0, v-1}$, which satisfies the following degree constraints:

$$
\begin{gathered}
n_{0}^{\prime}=n_{m 0}-n, \quad m_{i}^{\prime}(s)=n-1 \quad \text { for } i=\overline{0, v-1}, \\
\operatorname{Max}\left(n_{i}^{\prime}, m-1\right)=\operatorname{Max}\left(n_{m i}, \operatorname{Max}_{1 \leq k \leq i}\left(n_{k}+n_{i-k}^{\prime}\right)\right)-n .
\end{gathered}
$$

(ii) If (A1) holds and $n_{m 0} \geq 2 n$, then it is possible to build infinitely many proper rational functions of the form

$$
Q(s)=\frac{\sum_{l=0}^{v-1}\left[S_{l}(s)-\Lambda_{0}(s) A_{0}(s)\right] e^{-l h s}}{\sum_{l=k}^{v-1}\left[R_{l}(s)+\Lambda_{0}(s) B_{0}(s)\right] e^{-l h s}},
$$

with existing polynomial solution pairs $\left(R_{i}(s)-\Lambda_{0}(s) A_{0}(s), S_{i}(s)+\Lambda_{0}(s) B_{0}(s)\right)$ verifying (2.9) provided that $\left(R_{i}(s), S_{i}(s)\right)$ are also solutions to (2.9), where $\Lambda_{0}(s)=\lambda_{0}$ is any real scalar (i.e., any polynomial of zero degree) if $n>m$ and $\Lambda_{0}(s)$ is any arbitrary polynomial of arbitrary degree otherwise.

If $n_{m 0}=2 n-1$, then (2.12) is realizable for $\Lambda_{0}(s)=0$ if $n>m$ and with arbitrary $\Lambda_{0}(s)$ if $n=m$.

(iii) Assume that the controller transfer function (2.4) takes the subsequent specific form if Assumptions (A1) and (A3) hold with A(s) being strictly Hurwitzian:

$$
\begin{aligned}
K_{v}(s) & =\frac{S(s)}{R(s)} \\
& =\frac{\sum_{l=0}^{v-1}\left[S_{l}(s)-\Lambda_{0}(s) A_{0}(s)\right] e^{-l h s}}{\sum_{l=k}^{v-1}\left[R_{l}(s)+\Lambda_{0}(s) B_{0}(s)\right] e^{-l h s}+R_{v}(s)} \\
& =\frac{\sum_{i=0}^{v} S_{i}^{\prime}(s) e^{-i h s}}{\sum_{i=0}^{v-1} R_{i}^{\prime}(s) e^{-i h s}+R_{v}(s)} \\
& =\frac{D_{v}(s)\left(\sum_{i=0}^{v} \sum_{l=0}^{m_{i}^{\prime}} s_{i \ell}^{\prime} s^{l} e^{-i h s}\right)}{D_{v}(s)\left(\sum_{i=0}^{v-1} \sum_{l=0}^{r_{i}^{\prime}} r_{i \ell}^{\prime} s^{l} e^{-i h s}\right)+N_{v}(s) e^{-v h s}},
\end{aligned}
$$

where $\left(R_{i}(s), S_{i}(s)\right)$ are pairs of polynomials being any solutions to (2.9), $i=\overline{0, v-1} ; \Lambda_{0}(s)$ is chosen according to item (ii); $S_{v}(s)$ is an arbitrary polynomial of degree not exceeding $(n-1)$; and

$$
\begin{aligned}
R_{v}(s)=\frac{N_{v}(s)}{D_{v}(s)} & \\
=\frac{1}{A(s)} \times\left(\sum _ { l = v } ^ { v + q } \left[\hat{A}_{m l}(s)-\right.\right. & \sum_{i=\operatorname{Max}(v \cdot l-v+1)}^{\operatorname{Min}(l, q)} A_{i}(s) R_{l-i}(s) \\
& \left.\left.\left.+\sum_{i=\operatorname{Max}(v \cdot l-v)}^{\operatorname{Min}(l, q)} B_{i}(s) S_{l-i}(s)\right) e^{-(l-v) h s}\right]\right) .
\end{aligned}
$$


Then, the closed-loop spectrum satisfies $A(s) R(s)+B(s) S(s)=\hat{A}_{m}^{*}(s)=\sum_{i=0}^{v-1} \hat{A}_{m i}(s) e^{-i h s}$ with the closed loop being stable with poles in $\hat{A}_{m}^{*}(s)=0$ providing a closed-loop stable cancellation of the plant poles provided that $\hat{A}_{m}^{*}(s)=\sum_{l=0}^{v-1} A_{m l}(s) e^{-l h s}$ is a strictly Hurwitzian quasipolynomial satisfying $n_{m 0} \geq 2 n-1$.

(iv) If (A1) holds, $n_{m 0} \geq 2 n-1$, and the controller is simplified to have a transfer function $K_{v}^{*}(s)=Q(s)$ (i.e., $R_{v}(s)$ and $S_{v}(s)$ are zeroed), then the closed-loop spectrum is set to the zeros of

$$
\begin{aligned}
\hat{A}_{m}(s) & =\sum_{l=0}^{v+q} A_{m l}(s) e^{-l h s} \\
& =\left\{\hat{A}_{m}^{*}(s)\right\}+\left\{\sum_{\ell=v}^{v+q} \sum_{i=\operatorname{Max}(0, l-v)}^{\operatorname{Min}(l, q)}\left[A_{i}(s) R_{l-i}(s)+B_{i}(s) S_{l-i}(s)\right] e^{-l h s}\right\}
\end{aligned}
$$

without cancellations of the plant poles.

(v) If (A2) replaces (A1), then items (i)-(iv) hold for almost all $h \geq 0$.

Remark 2.2. Note that if $A(s)$ and $B(s)$ (resp., $A_{0}(s)$ and $B_{0}(s)$ ) have common stable zeros, then the controller synthesis problem is solvable if the objective spectrum contains those factors.

Remark 2.3. Note that the objective of the term $R_{v}(s)$ is to remove the unsuitable term

$$
\Delta \hat{A}_{m}^{*}(s)=\sum_{\ell=v}^{v+q} \sum_{i=\operatorname{Max}(0, l-v)}^{\operatorname{Min}(l, q)}\left[A_{i}(s) R_{l-i}(s)+B_{i}(s) S_{l-i}(s)\right] e^{-l h s}
$$

from the closed-loop spectrum. If the simplified controller of Theorem 2.1(iv) is used, then this term is not removed and the spectrum cannot be arbitrarily assigned.

Remark 2.4. Since $R(s)$ is, in general, a rational complex function, the basic controller synthesis (2.8) adopts the particular form

$$
A(s) \frac{\bar{R}(s)}{A(s)}+B(s) S(s)=\hat{A}_{m}(s)=\left\{\hat{A}_{m}^{*}(s)\right\}+\left\{\Delta \hat{A}_{m}^{*}(s)\right\}
$$

with $\bar{R}(s)=\hat{A}_{m}(s)-B(s) S(s)$ being a polynomial and $R(s)=\bar{R}(s) / A(s)$. After canceling the unsuitable closed-loop dynamics associated with $\Delta \hat{A}_{m}^{*}(s)$, the resulting closed-loop characteristic quasipolynomial arising while solving (2.8) reduces to the suitable dynamics given by the closed-loop equation

$$
A(s) R(s)+B(s) S(s)=\hat{A}_{m}^{*}(s) A(s),
$$

where $R(s)=\sum_{i=0}^{v} R_{i}(s) e^{-i h s}$ and $S(s)=\sum_{i=0}^{v} S_{i}(s) e^{-i h s}$ with $R_{v}(s)$ being rational. The polynomial $A(s)$ also generates zeros of the closed-loop transfer function obtained via the feed-forward controller (2.13) with $D_{\nu}(s)=A(s)$ so that $A(s)$ becomes a closed-loop stable cancellation. 
If the transfer function $P(s)$ of (2.1) is biproper, that is, $n=m$, then it is possible to modify the controller $(2.13)$ by setting $R_{v}(s)$ as a polynomial of degree at most $(n-1)$ and $S_{v}(s)$ as a rational function to remove the unsuitable remaining term in (2.14) from the spectrum. This will result in a closed-loop characteristic equation defined by $A(s) R(s)+$ $B(s) S(s)=\hat{A}_{m}^{*}(s) B(s)$. Section 3 will discuss the situation when the controller of transfer function structure (2.13) is replaced with the lower complexity controller of a transfer function structure (2.12), being a quotient of quasipolynomials. It will be proved that for sufficiently small sizes of the matrices defining the delayed dynamics in (2.1) the spectrum assignment objective is solved approximately in this way.

Remark 2.5. If (2.1) is not stable but is stabilizable, that is, $\operatorname{rank}\left[s I-\sum_{i=0}^{q_{0}} \overline{\mathbf{A}}_{i} e^{-i h s}: b\right]=$ $n$ for all complex $s$ with $\operatorname{Re} s \geq 0$, then it may be first stabilized with some stabilizing controller (which always exists) and then a prescribed closed-loop spectrum to the above obtained stable system might be assigned with a controller of transfer function structure (2.13). For instance, assume that the pair $\left(\overline{\mathbf{A}}_{\mathbf{0}}, b\right)$ is controllable, that is, the delay-free system (2.1) obtained after removing all the delayed dynamics is controllable. Thus, the state-feedback control law $u_{1}(t)=k^{T} x(t)$, where $k_{i}=c_{i}^{*}-c_{i}(i=\overline{0, n-1})$ sets the closedloop spectrum of such a delay-free system to the zeros of $c^{*}(s)=s^{n}+\sum_{i=0}^{n-1} c_{i}^{*} s^{i}$ provided that $\overline{\mathbf{A}}_{\mathbf{0}}$ is in a matrix in canonical controllable companion form with the last row being $\left(-c_{0},-c_{1}, \ldots,-c_{n-1}\right)$. Now, the unforced delayed system obtained with an input signal $u(t)=u_{1}(t)$ remains asymptotically stable from Nyquist's stability criterion [22] if

$$
1>\frac{q_{0} \bar{a}}{\left(\alpha_{c}^{*}\right)^{n}} \geq \sum_{i=1}^{q_{0}}\left(\left\|\frac{\overline{\mathbf{A}}_{i} e^{-i h s}}{c^{*}(s)}\right\|_{\infty}\right),
$$

where the above norm applies for rational stable transfer matrices in $\mathbb{R} \mathbb{U}_{\infty}, \alpha_{c}^{*}$ is the absolute value of the stability abscissa of $\left(\overline{\mathbf{A}}_{\mathbf{0}}+b k^{T}\right)$, that is, $\left(-\alpha_{c}^{*}\right)$ is the real part of the zero of $c^{*}(s)$ being closer to the imaginary axis, and $\bar{a} \geq \operatorname{Max}_{1 \leq i \leq q_{0}}\left(\left\|\overline{\mathbf{A}}_{i}\right\|_{2}\right)$. Note that if $\bar{a}$ is large, stability is maintained by correspondingly increasing $\alpha_{c}^{*}$. As a result, $\operatorname{det}\left(s I-\overline{\mathbf{A}}_{\mathbf{0}}-\right.$ $\left.b k^{T}-\sum_{i=1}^{n} \overline{\mathbf{A}}_{i} e^{-i h s}\right)$ has all its zeros in Res $<0$. Now, the closed-loop spectrum assignation method may be applied to the above stabilized system by generating an extra control signal $u_{2}(t)$ from a controller within the transfer function structure (2.13) so that the control law

$$
u(t)=u_{1}(t)+u_{2}(t)=\left(k+\frac{S(D)}{R(D)}\right) c^{T} x(t)+\frac{S(D)}{R(D)} y^{*}(t)
$$

stabilizes system (2.1) whereas it assigns its closed-loop spectrum to the zeros of $\hat{A}_{m}^{*}(s)$ provided that the controller of transfer function (2.13) is used with

$$
D_{\nu}(s)=A(s)=\operatorname{det}\left(s I-\overline{\mathbf{A}}_{\mathbf{0}}-b k^{T}-\sum_{i=1}^{n} \overline{\mathbf{A}}_{i} e^{-i h s}\right)
$$

being canceled by stable zeros in the closed-loop transfer function. The above technique may be easily simplified by first assigning only the unstable and critically stable zeros of $\operatorname{det}\left(s I-\overline{\mathbf{A}}_{0}\right)$, if any, via the control signal $u_{1}(t)$ without removal of the stable ones 
provided that the pair $\left(\overline{\mathbf{A}}_{\mathbf{0}}, b\right)$ is stabilizable. If the poles of (2.1) satisfying Re $s \geq 0$ may be separated in a factor $A^{-}(s)$ from $A(s)$, then an output-feedback stabilizing controller may be alternatively derived from Youla's parametrization [23] to synthesize a stabilizing input $u_{1}(t)$ for system (2.1) before applying the proposed assignment method via the control signal $u_{2}(t)$.

\section{Approximate spectrum assignment and robust stability for sufficiently small amounts of delayed dynamics}

Now, assume for convenience that $\overline{\mathbf{A}}_{\mathbf{0}}=\mathbf{A}_{\mathbf{0}}, \overline{\mathbf{A}}_{i}=\rho \mathbf{A}_{i}\left(i=\overline{1, q_{0}}\right)$ with $\rho \in \mathbb{R}$. Note that (2.1)-(2.2) is free of delayed dynamics if $\rho=0$. The respective unforced delay-free particular systems are defined by matrices $\mathbf{A}_{\mathbf{0}}$ and $\left(\mathbf{A}_{\mathbf{0}}+\rho \sum_{i=1}^{q} \mathbf{A}_{i}\right)$, respectively. It is now investigated when the closed-loop spectrum may be approximately assigned while maintaining the closed-loop stability by using a low-complexity controller of transfer function $K_{v}^{*}(s)=Q(s)$ with $Q(s)$ defined in (2.12). In this case, the rational function $R_{v}(s)$ and $S_{v}(s)$ are both zeroed at the expense of losing, in general, the property of exact spectrum assignment but with the advantage that neither of the plant poles or zeros are canceled in the closed-loop system so that (A3) is not required. It is proved that for sufficiently small $|\rho|$ (i.e., for sufficiently small delayed dynamics), the spectrum may be approximately assigned and the closed-loop stability may be achieved.

3.1. Mathematical results. The above properties are summarized in the next result which is proved in Appendix B.

Theorem 3.1 (approximate spectrum assignment and closed-loop stability for sufficiently small delayed dynamics). Assume that a low-complexity controller of the transfer function $K_{v}^{*}(s)=Q(s)$ is used for a system (2.1) satisfying Assumptions (A1)-(A2). Thus, the closed-loop spectrum consists of the zeros of $\hat{A}_{m}(s)$ defined in (2.14) for arbitrary prescribed $\hat{A}_{m}^{*}(s)$. Furthermore, there is $\rho_{0}>0$ such that the closed-loop spectrum remains stable for all

$\rho \in\left[-\rho_{0}, \rho_{0}\right]$ and arbitrarily close to the zeros of $\hat{A}_{m}^{*}(s)$ for a sufficiently small $\rho_{0}$ provided that $\hat{A}_{m}^{*}(s)$ is strictly Hurwitzian.

Corollary 3.2. Assume that $\hat{A}_{m}^{*}(s)$ is strictly Hurwitzian with stability abscissa $\left(-\sigma_{0}\right)<0$. Thus, for any $\sigma \in\left(0, \sigma_{0}\right)$, there always exists $\rho_{0}>0$ such that the closed-loop spectrum has stability abscissa $-\sigma_{c} \leq-\sigma<0$ for all $\rho \in\left[-\rho_{0}, \rho_{0}\right]$.

The proof of Corollary 3.2 is also given in Appendix B.

Remark 3.3 (practical use of Theorem 3.1 or Corollary 3.2). The computation of an available upper bound $\rho_{0}>0$ to apply Theorem 3.1 and Corollary 3.2 may be performed numerically from the sufficiency-type conditions derived in their respective proofs. This may be performed by starting with values $\rho=\rho_{0}=0$ and by increasing it in small positive amounts $\Delta \rho$ in case (B.5), or (B.6), in Appendix B holds if Theorem 3.1 is used, or, respectively, (B.7) holds if Corollary 3.2 is used. An available valid upper bound may also be calculated graphically by representing some of the various related conditions (see the above-mentioned formulas) by the intersection in the first quadrant of two curves of the type $\rho_{0}=\operatorname{Max}_{\rho \geq 0} \rho: G(\rho)=\rho<F^{-1}(\rho)$. The derivation of an explicit analytic formula is 
unfeasible in the more general cases because of the nonlinear nature of the respective sufficiency-type conditions.

The subsequent result is proved in Appendix C. It is referred to the robust stability problem in the presence of plant unmodeled dynamics and/or parametrical uncertainties if either the nominal controller or its associate low-complexity one is kept in operation.

Theorem 3.4 (robust stability theorem under plant uncertainties). Consider the plant (2.1)-(2.2) under Assumptions (A1)-(A3) together with the following additional ones.

(A4) The nominal (i.e., disturbance-free) connection is well posed (i.e., $1+K_{v}(\infty) P(\infty) \neq$ $0)$ and the nominal closed-loop system is stable.

(A5) The plant transfer function is perturbed from $P(s)$ to $(P(s)+\Delta(s))$ with $\Delta \in \mathbb{R} \mathbb{H}_{\infty}$. Thus, the following items hold:

(i) the resulting closed-loop system remains well posed and internally stable if and only if $\left\|K_{v}(s) \Delta(s) /\left(1+P(s) K_{v}(s)\right)\right\|_{\infty}<1$, where $K_{v}(s)$ is the nominal controller transfer function (2.13);

(ii) there are nominal controllers of transfer function (2.13), which keep the wellposedness of the feedback connection under the same assumptions as in (i) for all $\rho \in$ $\left[-\rho_{0}, \rho_{0}\right]$ and some sufficiently small $\rho_{0}>0$. If (A5) holds with the low-complexity controller of transfer function $K_{v}^{*}(s)=Q(s)$ of (2.12), then the well-posedness of the feedback connection holds if $\left\|K_{v}^{*}(s) \Delta(s) /\left(1+P(s) K_{v}^{*}(s)\right)\right\|_{\infty}<1$ for all $\rho \in\left[-\rho_{0}^{*}\right.$, $\left.\rho_{0}^{*}\right]$ for some sufficiently small $\rho_{0}^{*}>0$.

Remark 3.5. Note, for instance, that if $P(\infty)=-1 / K_{v}(\infty)$ with $K_{v}(\infty)$ being a constant nominal controller, then the feedback connection is not well posed since output or sensor measurement errors are transmitted through the loop by nonrealizable transfer functions [23]. If $d=0$, then $P(\infty)=0$ and the nominal feedback connection is always guaranteed to be well posed since Theorem 3.4(A) holds. The feedback well-posedness under plant disturbances and nominal controller is guaranteed if $\Delta(\infty) \neq-\left(P(\infty)+K_{v}^{-1}(\infty)\right)$ provided that the controller is biproper (since $K_{v}^{-1}(\infty) \neq 0$ ) and always guaranteed if it is strictly proper since (A) of Theorem 3.4 holds. This is guaranteed from its proof if $\Delta(s) \in \mathbb{R} \mathbb{M}_{\infty},\left\|K_{v}(s) \Delta(s) /\left(1+P(s) K_{v}(s)\right)\right\|_{\infty}<1$, and either $P(\infty) \neq-K_{v}^{-1}(\infty)$ with biproper stabilizing controller or $K_{v}(\infty)=0$ (i.e., the nominal controller is strictly proper).

3.2. Examples. Some examples are now given concerning the exact spectrum assignment of Section 2 and the approximate one of the current section.

Example 3.6. Assume the plant transfer function $P(s)=\left(s+2 e^{-h s}\right) /\left(s+1+\rho e^{-h s}\right)$. A physical problem described by this example for the unforced case is the (linearized) socalled Mackey-Glass equation. A more complex version would arise with $\rho$ being replaced by a (nonlinear) real monotone function of $\rho$. If such a function is nonmonotone, then the equation still possesses massive problems like, for instance, strange attractors, routes of chaos, as well as bifurcations of the periodic solutions which are phenomena caused by delays combined with nonlinearities. The problem in those general cases remains without being solved so that the attention is paid to the forced linear case according to the analysis and design philosophy proposed in this paper. If $|\rho|<1$, then 
the open-loop plant is globally asymptotically stable as deduced by simple inspection. The spectrum assignment method of Section 3 with $v=1$ is used for a designed suitable finite closed-loop spectrum given by the polynomial $\hat{A}_{m}^{*}(s)=0.1 s+2$. An obtained delaydependent particular controller transfer function (2.13) by using Theorem 2.1(iii) with one Diophantine equation of polynomials, a rational $R_{1}(s)$, and $\Lambda_{0}(s)=S_{1}(s)=0$ possess a transfer function $K_{1}(s)=-1.9\left(s+1+\rho e^{-h s}\right) /\left(2(s+1)+3.8 e^{-h s}\right)$. The closed-loop transfer function possesses in addition a zero-pole cancellation factor $A(s)=s+1+\rho e^{-h s}$ which is stable for $|\rho|<1$. If $\rho=2$, then a low-complexity controller of transfer function $K_{1}^{*}(s)=\left(1.9-0.2 e^{-h s}\right) /\left(0.2 e^{-h s}-2\right)$ assigns exactly the same stable closed-loop spectrum as above without any zero-pole closed-loop cancellation.

If now Theorem 2.1(iv) is applied for arbitrary $\rho$, the resulting controller is a pure gain $K_{1}^{*}=-0.95$ that yields a closed-loop spectrum defined by $\hat{A}_{m}(s)=0.1 s+2+(2 \rho-$ $3.8) e^{-h s}$ which contains unsuitable dynamics due to the use of a low-complexity controller. Since (A3) is not required because the low-complexity controller does not generate closed-loop cancellations, $\rho$ is not constrained a priori to guarantee the fulfillment of (A3). By examining the resulting spectrum, it is found from the small gain theorem that the closed-loop system is asymptotically stable if $\|(2 \rho-3.8) /(0.1 s+2)\|_{\infty}<1$ which is guaranteed if $\rho \in(-0.9,2.9)$. If now, the plant transfer function becomes $P(s)=$ $\left(s+2 \rho e^{-h s}\right) /\left(s+1+\rho e^{-h s}\right)$ (i.e., the delay contributions in both the numerator and denominator depend linearly on $\rho$ ), then the closed-loop asymptotic stability is guaranteed by using the same low-complexity pure-gain controller if $1.8|\rho|\|1 /(0.1 s+2)\|_{\infty}<1$, that is, for sufficiently small $|\rho|<1.227$, and the closed-loop poles are very close to the suited spectrum as $\rho$ tends to zero in the light of Theorem 3.1.

Example 3.7. Assume the plant transfer function $P(s)=\left(s+1+e^{-h s}\right) /\left(s^{2}+e^{-h s}\right)$. This example describes in the unforced case a harmonic oscillator with internal delay. In the forced case, since the zero is relevant to the dynamics, then the above transfer function describes an oscillator with internal delay in cascade with a derivative control (designed to improve the relative stability degree) involving also a delay. A general class of realizable controllers of transfer function (2.13) to assign the spectrum to the zeros of $\hat{A}_{m}^{*}(s)=s^{4}+4 s^{3}+8 s^{2}+8 s+4=0$ with $v=1$ is given by

$$
K_{1}(s)=\frac{\left[4(s+1)-\lambda_{0} s^{2}+S_{1}(s) e^{-h s}\right]\left(s^{2}+e^{-h s}\right)}{(s+2)^{2} s^{2}+\lambda_{0} s^{2}\left(s+1+e^{-h s}\right)-4(s+1) e^{-h s}-\left(s+1+e^{-h s}\right) S_{1}(s) e^{-h s}}
$$

after solving a Diophantine equation and adding the rational transfer function

$$
R_{1}(s)=-\frac{\left(s+1+e^{-h s}\right) S_{1}(s)+\left[(s+2)^{2}+4(s+1)\right]+\lambda_{0}\left(s+1-s^{2}\right)}{s^{2}+e^{-h s}}
$$

with $\lambda_{0}$ being any real number and $S_{1}(s)$ any polynomial of degree unity or zero (i.e., a constant). Note that the controller transfer function zeros include the plant poles which can include unstable factors dependent on the delays. Since the plant does not satisfy (A3), the closed-loop transfer function might include unstable cancellations. This drawback may be avoided either by first stabilizing the plant through the implementation of 
an extra control loop before assigning the spectrum (see Remark 2.5) or by using a lowcomplexity controller. The first approach modifies the original plant by including the stabilizing controller and then the design results similar to the above one leading to closedloop stable cancellations. The second approach avoids closed-loop cancellations so that it is applicable to unstable plants, while the spectrum is only approximately achievable and the closed-loop asymptotic stability maintained is for small contribution of the delays. To synthesize the low-complexity controller, consider the modified plant transfer function $P(s)=\left(s+1+\rho e^{-h s}\right) /\left(s^{2}+\rho e^{-h s}\right)$, where the amount of delayed dynamics may be zeroed by zeroing $\rho$. A low-complexity controller of transfer function $K_{1}^{*}(s)=4\left((s+1) /(s+2)^{2}\right)$ leads to a closed-loop spectrum given by $\hat{A}_{m}(s)=\hat{A}_{m}^{*}(s)+\left[4(s+1)+(s+2)^{2}\right] e^{-h s}$ with no zero-pole closed-loop cancellations, which is guaranteed to be stable if $|\rho|<0.5$ and approximately achievable as $\rho \rightarrow 0$.

Example 3.8. Assume the above plant transfer function includes an extra delay in the numerator and denominator resulting in $P(s)=\left(s+1+e^{-h s}+e^{-2 h s}\right) /\left(s^{2}+e^{-h s}+e^{-2 h s}\right)$. A realizable controller with $v=1$ within the general class of transfer functions (2.13) is $K_{1}(s)=4(s+1)\left(s^{2}+e^{-h s}+e^{-2 h s}\right) /\left(s^{2}(s+2)^{2}-4(s+1)\left(1+e^{-h s}\right) e^{-h s}\right)$ after zeroing the free design constant and polynomial of the general synthesis procedure.

\section{Conclusions}

This paper has dealt with the synthesis problem of pole-placement-based controllers for systems with point delays. The robust stability of the design has also been discussed. Special emphasis has been devoted to obtain the set of proper controllers and to the achievement of prescribed (finite or infinite) closed-loop spectrum of the designer's choice. Generally speaking, the arbitrary spectrum assignment is achievable under rather weak conditions for controllers of sufficiently high complexity in their delayed dynamics. If such a complexity is reduced under an appropriate threshold, then the arbitrary spectrum assignment becomes lost but it still remains approximately achievable with an approximation degree depending on the above-mentioned threshold. However, the robust stability property still holds for certain degrees of tolerance to plant uncertainties and amounts of nominal delayed dynamics.

\section{Appendices}

\section{A. Proof of Theorem 2.1}

To shorten the proof of item (i), it is convenient to look first for a particular solution and then construct infinitely many others from the found solution as stated in (i). Note that (2.9) may be seen as a set of $v$ coupled Diophantine equations which may be sequentially solved from $i=0$ to $i=v-1$ since (A1) implies (A2). A solution exists with the choice of degrees $n_{m 0} \geq 2 n-1 ; n_{0}^{\prime}=n_{m 0}-n \geq n-1 ; m_{i}^{\prime}=n-1$ for $i=\overline{0, v-1}$; and $\operatorname{Max}\left(n_{i}^{\prime}, m-\right.$ $1)=\operatorname{Max}\left(n_{m i}, \operatorname{Max}_{1 \leq k \leq i}\left(n_{k}+n_{i-k}^{\prime}\right)\right)-n$ for $i=\overline{1, v-1}$. The solution corresponds directly with that associated with a compatible set of $N=v n+\left(n_{m 0}-n+1\right)+\sum_{i=1}^{v-1}\left(n_{i}^{\prime}+1\right)$ algebraic linear equations with a similar number of real unknowns (i.e., the set of coefficients of the pairs $\left.\left(R_{i}(s), S_{i}(s)\right), i=\overline{0, v-1}\right)$. 
To prove the existence of infinitely many solutions to the set of Diophantine equations (2.9), note that if $\Lambda(s)$ is any arbitrary polynomial of arbitrary degree, then

$$
A_{0}(s) R_{i}(s)+B_{0}(s) S_{i}(s)=A_{0}(s)\left[R_{i}(s)+\Lambda(s) B_{0}(s)\right]+B_{0}(s)\left[S_{i}(s)-\Lambda(s) A_{0}(s)\right]
$$

equalizes the right-hand side of (2.9) so that

$$
R_{i}^{\prime}(s)=R_{i}(s)+\Lambda(s) B_{0}(s), \quad R_{i}^{\prime}(s)=S_{i}(s)-\Lambda(s) A_{0}(s) \quad \text { for } i=\overline{0, v-1}
$$

is also a solution for (2.9) with $\Lambda(s)$ being of arbitrary degree and arbitrary coefficients. Thus, there are infinitely many solutions to (2.9) for each $\hat{A}_{m}(s)$ with the given degree constraints. Item (i) has been proved. Now, note that if $n_{m 0} \geq 2 n ; n_{0}^{\prime}=n_{m 0}-n \geq n ; m_{i}^{\prime}=$ $n-1$ for $i=\overline{0, v-1}$ so that the set of Diophantine equations are sequentially solvable as a compatible set of $N$ linear algebraic equations with $N$ real solutions, then, item (i) follows by taking $\Lambda(s)$ as a real scalar if $n>m$ or any arbitrary polynomial otherwise since $Q(s)$ in (2.12) is realizable under the above constraints. For the case $n_{m 0}=2 n-1$, the proof is also direct by checking polynomial degrees in $Q(s)$ in (2.12), and item (ii) has been proved with $\Lambda_{0}(s)=\Lambda(s)$ in (A.2). Item (iii) follows by direct calculation since the equalities (2.9)-(2.10) hold with a controller of transfer function (2.13). Item (iv) follows since (2.9) holds through a controller of transfer function (2.12) so long as $\Delta \hat{A}_{m}^{*}(s)$ is not zeroed; namely, (2.10) fails. Item (v) follows since (A2) implies (A1) for almost all finite values of the base delay and when it tends to infinity.

\section{B. Proof of Theorem 3.1}

The transfer function (2.2) of (2.1) satisfies the following relationships for any complex $s$ which is not an eigenvalue of $\mathbf{A}_{\mathbf{0}}$ :

$$
\begin{aligned}
A(\rho, s) & =\operatorname{det}\left(s I-\mathbf{A}_{\mathbf{0}}-\rho \sum_{i=1}^{q_{0}} \mathbf{A}_{i} e^{-i h s}\right) \\
& =A_{0}(s) \cdot \operatorname{det}\left(I-\rho\left(s I-\mathbf{A}_{\mathbf{0}}\right)^{-1} \sum_{i=1}^{q_{0}} \mathbf{A}_{i} e^{-i h s}\right) \\
& =A_{0}(s)-\rho \operatorname{tr}\left(\operatorname{Adj}\left(s I-\mathbf{A}_{\mathbf{0}}\right)\left(\sum_{i=1}^{q_{0}} \mathbf{A}_{i} e^{-i h s}\right)+O(\rho)\right) \\
& =A_{0}(s)+\rho A^{\prime}(\rho, s)=\sum_{i=0}^{n}\left[a_{0 i}+\rho a_{0 i}^{\prime}(\rho, s)\right] s^{i},
\end{aligned}
$$

where $f=O(\rho)$ if $\|f\| \leq K_{1}|\rho|+K_{2}$ for some real constants $K_{1,2} \geq 0$, and dependence of $A(\cdot)$ on $\rho$ has been made explicit in the notation for convenience. Thus, $A(\rho, s)=A_{0}(s)$ if $\rho=0$ and the coefficients of $A^{\prime}(\rho, s)$ depend, in general, on combined powers of $\rho$. 
Also, one gets directly by using a formal expansion of $\operatorname{Adj}(s I-\mathbf{A}(\rho, s))[11]$ for $\mathbf{A}(\rho, s)=$ $\mathbf{A}_{\mathbf{0}}+\rho \sum_{i=1}^{q_{0}} \mathbf{A}_{i} e^{-i h s}$,

$$
\begin{aligned}
B(\rho, s) & =c^{T} \operatorname{Adj}\left(s I-\mathbf{A}_{0}-\rho \sum_{i=1}^{q_{0}} \mathbf{A}_{i} e^{-i h s}\right) b+d A(s) \\
& =c^{T} \sum_{\mu=0}^{n-1}\left[\sum_{l=1}^{n-k}\left(a_{0 l}+\rho a_{0 l}^{\prime}(\rho)\right) s^{l-1}\right]\left(\mathbf{A}_{0}+\rho \sum_{i=1}^{q_{0}} \mathbf{A}_{i} e^{-i h s}\right)^{\mu} b+d A(\rho, s) \\
& =\left(B_{0}(s)+d A_{0}(s)\right)+\rho\left(B^{\prime \prime}(\rho, s)\right)+d \rho A^{\prime}(\rho, s)
\end{aligned}
$$

after using $A(\rho, s)=A_{0}(s)+\rho A^{\prime}(\rho, s)$ from (B.1) with

$$
B(\rho, s)_{\rho=0}=B_{0}(s)+d A_{0}(s)=c^{T}\left(\sum_{\mu=0}^{n-1} \sum_{l=0}^{n-k} a_{0 l} s^{l-1}\right) \mathbf{A}_{0}^{\mu} b+d \sum_{i=0}^{n-1} a_{0 i} s^{i}
$$

being of degree $n$ if $d \neq 0$ and $m \leq n-1$ if $d=0$. Now, note that $P(s)=\left(B_{0}(s)+\rho B^{\prime}(\rho, s)\right) /$ $\left(A_{0}(s)+\rho A^{\prime}(\rho, s)\right)$, where the coefficients of $B^{\prime}(\rho, s)$ and $A^{\prime}(\rho, s)$ depend, in general, on a set of powers of $\rho$. By comparing the above expression for $P(s)$ with that obtained from (2.3), it follows that all $A_{i}(s)$ and $B_{i}(s)$ may be rewritten as $A_{i}(\rho, s)=\rho A_{i}^{\prime}(\rho, s)$; $B_{i}(\rho, s)=\rho B_{i}^{\prime}(\rho, s)$ and thus $a_{i k}(\rho)=\rho a_{i k}^{\prime}(\rho) ; b_{i k}(\rho)=\rho b_{i k}^{\prime}(\rho)$ for $i \geq 1$. Then, the closedloop spectrum for any controller of reduced complexity of transfer function $K_{v}^{*}(s)=Q(s)$ becomes $A(s) R(s)+B(s) S(s)=\hat{A}_{m}^{*}(s)+\Delta \hat{A}_{m}^{*}(\rho, s)$, where

$$
\begin{gathered}
\Delta \hat{A}_{m}^{*}(\rho, s)=\rho \tilde{A}_{m}(\rho, s), \\
\tilde{A}_{m}(\rho, s)=\sum_{\ell=v}^{v+q} \sum_{i=\operatorname{Max}(0, l-v)}^{\operatorname{Min}(l, q)}\left[A_{i}^{\prime}(s) R_{l-i}(s)+B_{i}^{\prime}(s) S_{l-i}(s)\right] e^{-l h s}
\end{gathered}
$$

with $\hat{A}_{m}^{*}(s)$ being strictly Hurwitzian. From Rouché's theorem for zeros of complex functions (or from Nyquist's stability criterion) (see [22]) applied for any bounded open semicircle of arbitrarily large radius included in the right-half complex plane $\operatorname{Re} s \geq 0[19,22]$, the closed-loop spectrum is stable if $\left|\rho \tilde{A}_{m}(\rho, j \omega)\right|<\left|\hat{A}_{m}^{*}(j \omega)\right|$ for all $\omega \in \mathbb{R}_{0}^{+}=\mathbb{R}^{+} \cup\{0\}$. The above inequality is guaranteed if $\rho \in\left[-\rho_{0}, \rho_{0}\right]$ and

$$
\rho_{0} \operatorname{Sup}_{|\rho| \leq \rho_{0}}\left(\operatorname{Sup}_{\omega \in \mathbb{R}_{0}^{+}}\left(\left|\frac{\tilde{A}_{m}(\rho, j \omega)}{\hat{A}_{m}^{*}(j \omega)}\right|\right)\right)<1 .
$$

Such a $\rho_{0}>0$ always exists since $\tilde{A}_{m}(0, j \omega) \equiv 0$ from (B.1)-(B.2). Equation (B.5) holds if $\rho_{0}<\rho_{0}^{\prime-1}$, where

$$
\rho_{0}^{\prime}=\operatorname{Sup}_{z \in \mathbb{R}_{0}^{+}}\left(\operatorname{Sup}_{\omega \in \mathbb{R}_{0}^{+}}\left(\left|\frac{\tilde{A}_{m}(z, j \omega)}{\hat{A}_{m}^{*}(j \omega)}\right|\right)\right) \leq 1 \quad \text { or } \quad \rho_{0}^{\prime}=\operatorname{Max}\left(z \in \mathbb{R}_{0}^{+}:\left\|\tilde{H}_{z}\right\|_{\infty} \leq 1\right)
$$

with $\tilde{H}_{z}(s)=\tilde{A}_{m}(z, s) / \hat{A}_{m}^{*}(s)$ being an $\mathbb{R} \mathbb{T}_{\infty}$-Schur's function since $\hat{A}_{m}^{*}(s)$ is strictly Hurwitzian $[10,23]$. It has been proved that there exists $\rho_{0}>0$ such that the closed-loop 
spectrum is stable for all $\rho \in\left[-\rho_{0}, \rho_{0}\right]$ provided that $\hat{A}_{m}^{*}(s)$ is strictly Hurwitzian. On the other hand, the closed-loop spectrum satisfies $A_{m}(s) \equiv 0 \Leftrightarrow 1+\rho\left(\tilde{A}_{m}(\rho, s) / \hat{A}_{m}^{*}(s)\right)=0$ which is close to the zeros of $\hat{A}_{m}^{*}(s)$ as $|\rho| \rightarrow 0$ from the root locus technique. Therefore, the closed-loop spectrum $\hat{A}_{m}^{*}(s)=0$ is approximately achievable independent of the delay for arbitrarily small $|\rho|$, and Theorem 2.1 has been proved.

Proof of Corollary 3.2. Again from Rouché's theorem (or from Nyquist's stability criterion), $\hat{A}_{m}^{*}(s)$ and $\hat{A}_{m}(\rho, s)$ have the same number of zeros (i.e., none) on Re $s \geq-\sigma$ with $\sigma \in\left(0, \sigma_{0}\right)$ if $|\rho|\left|\tilde{A}_{m}(\rho, j \omega)\right|<\left|\hat{A}_{m}^{*}(j \omega)\right|$ for all $\omega \in \mathbb{R}_{0}^{+}$since $A_{m}^{*}(s)$ is strictly Hurwitzian for $\operatorname{Re} s \geq-\sigma_{0}$. Thus, the proof follows as in that of Theorem 2.1 to yield $\rho_{0}<\rho_{0}^{\prime-1}$ with

$$
\rho_{0}^{\prime}=\operatorname{Sup}_{z \in \mathbb{R}_{0}^{+}}\left(\operatorname{Sup}_{\omega \in \mathbb{R}_{0}^{+}}\left(\left|\frac{\tilde{A}_{m}(z,-\sigma+j \omega)}{\hat{A}_{m}^{*}(-\sigma+j \omega)}\right|\right)\right) \leq 1
$$

and there is some real $\sigma_{c} \geq \sigma$ such that all the zeros of $A_{m}(s)$ are in $\operatorname{Re} s \leq-\sigma_{c}$ for all $\rho \in\left[-\rho_{0}, \rho_{0}\right]$.

\section{Proof of Theorem 3.4}

(i) Sufficiency. The closed-loop characteristic equation is

$$
1+(P(s)+\Delta(s)) K_{v}(s)=0 \Longrightarrow A(s) R(s)+B(s) S(s)+A(s) S(s) \Delta(s)=0
$$

which is stable from Rouché's theorem if

$$
|A(j \omega) S(j \omega)|\left|\Delta_{B}(j \omega)\right|<|A(j \omega) R(j \omega)+B(j \omega) S(j \omega)|\left|\Delta_{A}(j \omega)\right|
$$

for all $\omega \in \mathbb{R}_{0}^{+}$with $\Delta(s)=\Delta_{B}(s) / \Delta_{A}(s)$ since $A(s) R(s)+B(s) S(s)=\hat{A}_{m}(s) A(s) \neq 0$ for Res $<0$. Note that (C.2) holds if $\left\|K_{v}(s) \Delta(s) /\left(1+P(s) K_{v}(s)\right)\right\|_{\infty}=\left\|A(s) S(s) \Delta(s) / \hat{A}_{m}(s)\right\|_{\infty}<$ 1. Also,

$$
1+(P(\infty)+\Delta(\infty)) K_{v}(\infty)=\left(1+P(\infty) K_{v}(\infty)\right)\left[1+\left(1+P(\infty) K_{v}(\infty)\right)^{-1} K_{v}(\infty) \Delta(\infty)\right] \neq 0
$$

if $1+P(\infty) K_{v}(\infty) \neq 0$ (i.e., the nominal feedback connection is well posed and $K_{v}(\infty) \Delta(\infty) /\left(1+P(\infty) K_{v}(\infty)\right) \neq-1$ if $\left.\left\|K_{v}(s) \Delta(s) /\left(1+P(s) K_{v}(s)\right)\right\|_{\infty}<1\right)$. Then, the current closed-loop system for the disturbed plant is well posed and internally stable since $(P(s)+\Delta(s)) K_{v}(s)$ has no unstable zero-pole cancellation, since $P(s) K_{v}(s)$ has no unstable zero-pole cancellation from Assumptions (A1) $-(\mathrm{A} 3)$ and $\Delta(s) \in \mathbb{R} \mathbb{T}_{\infty}$.

Necessity. For $\Delta(s) \in \mathbb{R}_{\infty}$ such that $\left\|K_{v}(s) \Delta(s) /\left(1+P(s) K_{v}(s)\right)\right\|_{\infty} \geq 1$, the Nyquist's plot encloses the critical point for some range of frequencies and then the closed-loop stability becomes lost. 
(ii) The characteristic equation of the closed-loop system for the connection of the disturbed plant with the nominal controller is obtained from (C.2):

$$
1+\frac{\rho \tilde{A}_{m}(\rho, s)+A(s) S(s) \Delta(s)}{\hat{A}_{m}^{*}(s)}=0
$$

with $\hat{A}_{m}^{*}(s)$ being strictly Hurwitzian. From Theorem 3.1, the closed-loop system is stable for all $\rho>0$ satisfying

$$
\left\|\rho \frac{\tilde{A}_{m}(\rho, s)}{\hat{A}_{m}^{*}(s)}+\frac{A(\rho, s) S(\rho, s) \Delta(s)}{\hat{A}_{m}^{*}(s)}\right\|_{\infty}<1
$$

Now, take any real constants $\gamma_{01} \in[0,1)$ and $\gamma_{02} \in\left[0,1-\gamma_{01}\right]$ and define real constants

$$
\begin{aligned}
& \rho_{01}=\operatorname{Max}\left(z \in \mathbb{R}_{0}^{+}:\left\|z \frac{\tilde{A}_{m}(z, s)}{\hat{A}_{m}^{*}(s)}\right\|_{\infty} \leq \gamma_{01}\right), \\
& \rho_{02}=\operatorname{Max}\left(z \in \mathbb{R}_{0}^{+}:\left\|\frac{A(z, s) S(z, s)}{\hat{A}_{m}^{*}(s)}\right\|_{\infty} \leq \gamma_{02}\right)
\end{aligned}
$$

which depend respectively on $\gamma_{01}$ and $\gamma_{02}$. Then, the closed-loop system is kept stable for all $\rho \in\left[-\rho_{0}, \rho_{0}\right]$ with $\rho_{0}=\operatorname{Min}\left(\rho_{01}, \rho_{02}\right)$ if $\|\Delta\|_{\infty}<\left(1-\gamma_{01}\right) / \gamma_{02}$ for the given $\gamma_{01}$ and $\gamma_{02}$. If the pair $\left(\rho_{0}, \delta_{0}\right) \in \mathbb{R} \times \mathbb{R}$ is defined as

$$
\left(\rho_{0}, \delta_{0}\right)=\left((\operatorname{Max}(\rho \geq 0), \operatorname{Max}(\delta \geq 0)):\left\|\rho \frac{\tilde{A}_{m}(\rho, s)}{\hat{A}_{m}^{*}(s)}+\frac{A(\rho, s) S(\rho, s) \Delta(s)}{\hat{A}_{m}^{*}(s)}\right\|_{\infty} \leq 1\right)
$$

then the closed-loop system is stable for all $\rho \in\left(-\rho_{0}, \rho_{0}\right)$ and $\delta \in\left[-\delta_{0}, \delta_{0}\right]$ or $\rho \in\left[-\rho_{0}, \rho_{0}\right]$ and $\delta \in\left(-\delta_{0}, \delta_{0}\right)$, irrespective of $\gamma_{01}$ and $\gamma_{02}$. A similar reasoning applies for the lowcomplexity controller of transfer function $K_{v}^{*}(s)$ under nominal well-posedness for sufficiently small $|\rho|$. The proof is completed.

\section{Acknowledgments}

The author is very grateful to Ministerio de Ciencia y Tecnologia (MCYT) for its partial support of this work via Project DPI 2003-00164 and to Universidad del País Vasco (UPV) for its support through Research Groups Grant 9/UPV/00I06.I06-15263/2003. He is also grateful to the reviewers for their interesting comments.

\section{References}

[1] C. F. Alastruey, Stabilizability of systems with two-point delays by using a generalized PD controller, Appl. Math. Lett. 11 (1998), no. 4, 39-42.

[2] C. F. Alastruey, M. de la Sen, and J. R. González de Mendívil, The stabilizability of integrodifferential systems with two distributed delays, Math. Comput. Modelling 21 (1995), no. 8, 85-94.

[3] A. Bellen, N. Guglielmi, and A. Ruehli, Methods for linear systems of circuit delay differential equations of neutral type, IEEE Trans. Circuits Syst. I 46 (1999), no. 1, 212-215. 
[4] T. A. Burton, Stability and Periodic Solutions of Ordinary and Functional-Differential Equations, Mathematics in Science and Engineering, vol. 178, Academic Press, Florida, 1985.

[5] C. Corduneanu and N. Luca, The stability of some feedback systems with delay, J. Math. Anal. Appl. 51 (1975), no. 2, 377-393.

[6] M. de la Sen, On some structures of stabilizing control laws for linear and time-invariant systems with bounded point delays and unmeasurable states, Internat. J. Control 59 (1994), no. 2, 529-541.

[7] Allocation of the poles of delayed systems related to those associated with their undelayed counterparts, Electron. Lett. 36 (2000), no. 4, 373-374.

[8] M. de la Sen and J. Jugo, Pole-placement controllers for linear systems with point delays, IMA J. Math. Control Inform. 13 (1996), no. 3, 223-249.

[9] M. de la Sen and N. Luo, On the uniform exponential stability of a wide class of linear time-delay systems, J. Math. Anal. Appl. 289 (2004), no. 2, 456-476.

[10] B. A. Francis, A Course in $H_{\infty}$ Control Theory, Lecture Notes in Control and Information Sciences, vol. 88, Springer-Verlag, Berlin, 1987.

[11] T. Kailath, Linear Systems, Prentice-Hall, New Jersey, 1980.

[12] E. W. Kamen, Linear systems with commensurate time delays: stability and stabilization independent of delay, IEEE Trans. Automat. Control AC-27 (1982), no. 2, 367-375.

[13] E. W. Kamen, P. Khargonekar, and A. Tannenbaum, Stabilization of time-delay systems using finite-dimensional compensators, IEEE Trans. Automat. Control AC-30 (1985), no. 1, 75-78.

[14] N. Luo and M. de la Sen, State feedback sliding mode control of a class of uncertain time delay systems, IEE Proc. D Control Theory and Applications 140 (1993), no. 4, 261-274.

[15] N. Luo, M. de la Sen, and J. Rodellar, Robust stabilization of a class of uncertain time delay systems in sliding mode, Internat. J. Robust Nonlinear Control 7 (1997), no. 1, 59-74.

[16] A. J. Manitius and A. J. Olbrot, Finite spectrum assignment problem for systems with delays, IEEE Trans. Automat. Control AC-24 (1979), no. 4, 541-552.

[17] S.-I. Niculescu, Delay Effects on Stability. A Robust Control Approach, Lecture Notes in Control and Information Sciences, vol. 269, Springer-Verlag, London, 2001.

[18] A. E. Ruehli, Equivalent circuit models for three-dimensional multiconductor systems, IEEE Trans. Microwave Theory Tech. 22 (1974), no. 3, 216-221.

[19] R. Schätzle, On the perturbation of the zeros of complex polynomials, IMA J. Numer. Anal. 20 (2000), no. 2, 185-202.

[20] J.-C. Shen, B.-S. Chen, and F.-C. Kung, Memory-less stabilization of uncertain dynamic delay systems: Riccati equation approach, IEEE Trans. Automat. Control AC-36 (1991), no. 5, 638640.

[21] K. K. Shyu and J. J. Yan, Robust stability of uncertain time-delay systems and its stabilization by variable structure control, Internat. J. Control 57 (1993), no. 1, 237-246.

[22] A. D. Wunsch, Complex Variables with Applications, Addison-Wesley Publishing, Massachusetts, 1994.

[23] K. Zhou and J. C. Doyle, Essentials of Robust Control, Prentice Hall, New Jersey, 1998.

M. de la Sen: Departamento de Ingeniería de Sistemas y Automática, Instituto de Investigación y Desarrollo de Procesos (IIDP), Facultad de Ciencias, Universidad del País Vasco, Campus de Bizkais, Aptdo. 644, 48080 Bilbao, Spain

E-mail address: wepdepam@lg.ehu.es 